of these compounds (17). It is therefore possible that cancer may result from synergistic interactions between normal cellular UV light, bacteria-produced UV emissions and pollutants such as PAHs.

The suggested hypothesis would help explain why cancer is not infectious in the normal sense, since it implies that the UV produced by bacteria growing within the cell would operate as a 'cancer switch' which would also be influenced by both hereditary and environmental factors. The internal production of UV might be one of many ways by which intracellular bacteria might influence the operation of such a 'switch'.

Of course, 'the devil lies in the detail' and the main problem with this hypothesis relates to dose-effect. That is would sufficient UV be produced by intracellular bacteria to effect DNA mutagenesis, bearing in mind that any UV emissions would be subject to adsorption by cellular contents, including membranes?

Should the present hypothesis be correct, then vaccines might be developed to prevent the growth of intracellular bacteria. Alternatively, other agents might be found which could prevent such bacteria from emitting UV, or counteract its mutagenic effects. Such agents would be invaluable in preventing both the development or spread of cancer.

\section{Milton Wainwright}

Department of Molecular Biology and Biotechnology, University of Sheffield, Sheffield, S10 2TN, UK.

Tel: +441142224410 .

Fax: +44 1142728697

e-mail: M.Wainwright@shef.ac.uk

1. Wainwright, M., Killham, K., Russell, C. \& Grayston, S. J. (1997). Partial evidence for the existence of mitogenetic radiation. Microbiology 143, 1-3. 2. Wainwright, M. (1997). When heresies collide extreme bacterial pleomorphism and the cancer germ. Microbiology 144, 595-596.

3. Wainwright, M. (1995). The return of the cancer germ Soc Gen Microbiol Q 22,48-50.

4. Cantwell, A. R. (1990). The Cancer Microbe. Los Angeles: Aries Press.

5. Hess, D. J. (1997). Can Bacteria Cause Cancer? New York: New York University Press.

6. Eidt, S. \& Stolte, M. (1993). Helicobacter pylori and gastric malignancy. Zentbl Bakteriol 280, 137-143.

7. Nightingale, T. E. \& Gruber, J. (1994). Helicobacter and human cancer. J Natl Cancer Inst 86,1505-1509.

8. Franklin, C. L., Beckwith, C. S., Livingston, R. S., Riley, L. K., Gibson, S. V., Besch-Williford, C. L. \& Hook, R. R. (1996). Isolation of a novel Helicobacter species, Helicobacter cholecystus sp. nov., from the gallbladders of Syrian hamsters with cholangiofibrosis and centrilobular pancreatitis. J Clin Microbiol 34, 2952-2958.

9. Ohshima, H. \& Bartsch, H. (1994). Chronic infections and inflammatory processes as cancer risk factors: possible role of nitric oxide in carcinogenesis. Mutat Res 305, 253-264.

10. Chan, P. J., Seraj, I. M., Kalugdan, T. H. \& King, A. (1996). Prevalence of mycoplasma conserved DNA in malignant ovarian cancer detected using PCR-ELISA. Gynecol Oncol 63, 258-260.

11. Shen, X., Liu, F. \& Li, X. Y. (1993). Experimental study on photocount statistics of the ultraweak photon emission from some living organisms. Experientia 49, 291-295.

12. Tilbury, R. N. \& Quickenden, T. I. (1988) Spectral and time dependence studies of the ultraweak bioluminescence emitted by the bacterium Escherichia coli. Photochem Photobiol 47, 145-150.

13. Quickenden, T. I. \& Tilbury, R. N. (1983). Growth dependent luminescence from cultures of normal and respiratory deficient Saccharomyces cerevisiae. Photochem Photobiol 37, 337-344.

14. Kielbassa, C., Roza, L. \& Epe, B. (1997). Wave length dependence of oxidative DNA damage induced by UV and visible light. Carcinogenesis 18, 811-816.

15. Illingworth, J. E. (1986). The relationship between ultraviolet radiation and epithelial cancer Med Hypotheses 19, 155-160.

16. Morris, J. J. \& Seifter, E. (1992). The role of aromatic hydrocarbons in the genesis of breast cancer, Med Hypotheses 38, 177-184.

17. Arfsten, D. P., Schaeffer, D. J. \& Mulveny, D. C. (1996). The effects of near ultraviolet radiation on the toxic effects of polycyclic aromatic hydrocarbons in animals and plants: A review. Ecotoxicol Environ Saf 33, 1-24.

\section{Reviewer's Comments}

At first sight, this hypothesis appears unlikely. However, if it is an established fact that micro-organisms do produce UV light, then it is worth considering.

One possible way of testing this hypothesis is to look at the profile of mutations induced in a gene such as p53 in tumour cells. Skin cancers show significant levels of mutations at adjacent pyrimidines indicative of UV-damage. If this was shown to be the case for internal tumours this would suggest UV-damage at di-pyrimidine sites, a finding that would strongly support the hypothesis. However, the prevailing data do not show this, certainly not at significant levels. For example, lung tumours, associated with smoking, most often show transversions at $\mathrm{G}$ residues, consistent with exposure to PAHs. It may, however, be worth closely examining the mutation data for a variety of internal tumours to determine if UV-associated mutations occur as rare events (particularly in non-smokers).

One must also consider the wavelength of UV light produced by these organisms. UV-B and UV-C will produce pyrimidine dimers, whilst UV-A will not. Much less is known about the mutational effects of UV-A which may induce DNA damage indirectly through radical formation and not at dipyrimidines.

In summary, this is an interesting hypothesis, which while it might be unlikely, is not outside the bounds of possibility.

\section{Nigel J. Jones}

School of Biological Sciences, University of Liverpool

\section{Intracellular location of mycoplasmas}

Mycoplasmas associated with animals have generally been regarded as extracellular and their specific attachment to the surface of eukaryotic cells has been widely reported. However, in recent years evidence has accumulated that certain species, including Mycoplasma fermentans, Mycoplasma genitalium and Mycoplasma hominis, may have an intracellular location, and Mycoplasma penetrans, isolated from the urine of AIDS patients, has been shown to penetrate a wide range of cultured animal cells (1). The ability of mycoplasmas to survive within host cells is significant and might help explain the chronic nature of many mycoplasma infections and the persistence of asymptomatic carriers. Furthermore, their survival within professional phagocytic cells might lead to dissemination within the host.

$M$. fermentans has been implicated in human diseases affecting diverse body tissues. We have obtained electron micrographs of M. fermentans (PG18) with human polymorphonuclear leukocytes (PMNL) which show mycoplasmas clustered within phagosomes and also single mycoplasma cells apparently free within the cytoplasm (Fig. 1). There is an apparent interaction between the cytoplasm and these intracytoplasmic mycoplasmas that leads to the formation of an electron-dense layer completely enclosing the mycoplasma cell and approximately $30 \mathrm{~nm}$ in thickness. This electron-dense layer may be surrounded by a membrane. The frequency with which intracytoplasmic mycoplasmas were seen was greater where mycoplasma cells were non-opsonized than when non-specifically opsonized by incubation for $45 \mathrm{~min}$ in $10 \%$ human serum. In contrast, when mycoplasma cells were opsonized, intracellular mycoplasmas were almost always internalized within phagolysosomes.

It has been argued that in electron microscopic studies the appearance of mycoplasmas within cells might be artefactual and due to the presence of the mycoplasmas within invaginations of the cell membrane. Thus, Taylor-Robinson et al. (2) claimed that unequivocal evidence of the intracellular location of mycoplasmas required specific staining of both the mycoplasma and host cell surface. This was achieved in their study using gold-labelled anti-mycoplasma antibody and ruthenium red, which bound to the exposed polysaccharide surface of both mycoplasma and eukaryotic cell surfaces, but not to the membranes of cell vacuoles. Jensen et al. (3) found ruthenium red staining of the Vero cell membrane to be weak and of little value in confirming the apparent intracellular location of M. genitalium cells. They argued that where mycoplasma cells appeared close 